https://helda.helsinki.fi

\title{
An alternative capacity in metric measure spaces
}

\section{Martio, Olli}

$2021-10$

Martio , O 2021 , ' An alternative capacity in metric measure spaces ' , Journal of pÿmathematical sciences, vol. 258 , no. 3 , pp. 303312 . https://doi.org/10.1007/s10958-021-05548-3

http://hdl.handle.net/10138/339553

https://doi.org/10.1007/s10958-021-05548-3

unspecified

Downloaded from Helda, University of Helsinki institutional repository.

This is an electronic reprint of the original article.

This reprint may differ from the original in pagination and typographic detail.

Please cite the original version. 


\title{
An alternative capacity in metric measure spaces
}

\section{Olli Martio}

\author{
Dedicated to Vladimir Gutlyanskii on his $80^{\text {th }}$ birthday
}

\begin{abstract}
A new condenser capacity $\operatorname{Cap}_{\mathrm{p}}^{\mathrm{M}}(E, G)$ is introduced as an alternative to the classical Dirichlet capacity in a metric measure space $X$. For $p>1$, it coincides with the $M_{p}$-modulus of the curve family $\Gamma(E, G)$ joining $\partial G$ to an arbitrary set $E \subset G$ and, for $p=1$, it lies between $A M_{1}(\Gamma(E, G))$ and $M_{1}(\Gamma(E, G))$. Moreover, the $\operatorname{Cap}_{\mathrm{p}}^{\mathrm{M}}(E, G)$-capacity has good measure theoretic regularity properties with respect to the set $E$. The $\operatorname{Cap}_{\mathrm{p}}^{\mathrm{M}}(E, G)$-capacity uses Lipschitz functions and their upper gradients. The doubling property of the measure $\mu$ and Poincare inequalities in $X$ are not needed.
\end{abstract}

Keywords. Capacity, $M$ - and $A M$-moduli.

\section{Introduction}

The Dirichlet $p$-capacity $\operatorname{cap}_{p}(E, G)$ of a condenser $(E, G)$, developed by $\mathrm{G}$. Choquet, is the most commonly used capacity in analysis. The modulus of a curve family offers an alternative approach to capacity. In a metric measure space $X$, curve families play a more central role than in $\mathbb{R}^{n}$ since the Fubini theorem is not available in $X$. For example, in $X$, the modulus method is used to construct the so-called Newtonian spaces, which have many properties common to the first order Sobolev spaces in $\mathbb{R}^{n}$. The constructions require that the metric space $X$ should be a so-called good metric space, i.e. the measure $\mu$ in $X$ be doubling and $X$ support the Poincare inequality in addition to various topological properties, see [2], [7] and [13].

The purpose of this paper is to introduce an alternative capacity, the $\mathrm{Cap}_{\mathrm{p}}^{\mathrm{M}}$-capacity, which is directly connected to the $M_{p}$-modulus for $p>1$ and uses neither the doubling property nor the Poincaré inequalities. For $p=1$, the Cap $\mathrm{p}_{\mathrm{p}}^{\mathrm{M}}$-capacity lies between the $A M_{1^{-}}$and $M_{1}$-moduli.

The Cap $\mathrm{p}_{\mathrm{p}}^{\mathrm{M}}$-capacity, $p>1$, offers a more straightforward approach to the classical Dirichlet capacity cap $_{p}$, and their equivalence is considered in Section 4.

\section{2. $\quad M_{p^{-}}$and $A M_{p^{-}}$moduli}

Let $(X, d)$ be a metric space equipped by a Borel regular measure $\mu$ which is finite on compact sets. We also assume that $X$ is proper, i.e. bounded closed sets are compact. From this, it follows that $X$ is complete.

A continuous mapping $\gamma:[a, b] \rightarrow X$ is called a curve. We say that a curve $\gamma$ is a path if it has a finite and non-zero total length; in this case, we parametrize $\gamma$ by its arclength. The locus of $\gamma$ is defined as $\gamma([0, \ell])$ and denoted by $\langle\gamma\rangle$, and the length of $\gamma$ by $\ell(\gamma)$.

Let $\Gamma$ be a family of paths in $X$. A non-negative Borel function $\rho$ is $M$-admissible, or simply admissible, for $\Gamma$ if

$$
\int_{\gamma} \rho d s \geq 1
$$

Translated from Ukrains'kiı̆ Matematychnyı̆ Visnyk, Vol. 18, No. 2, pp. 196-208, April-June, 2021.

Original article submitted June 12, 2021 


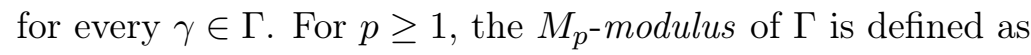

$$
M_{p}(\Gamma)=\inf \int_{X} \rho^{p} d \mu,
$$

where the infimum is taken over all admissible functions $\rho$.

A sequence of non-negative Borel functions $\rho_{i}, i=1,2, \ldots$, is $A M$-admissible, or simply admissible, for $\Gamma$ if

$$
\liminf _{i \rightarrow \infty} \int_{\gamma} \rho_{i} d s \geq 1
$$

for every $\gamma \in \Gamma$. The approximation modulus $\Gamma$ is defined as

$$
A M_{p}(\Gamma)=\inf _{\left(\rho_{i}\right)}\left\{\liminf _{i \rightarrow \infty} \int_{X} \rho_{i}^{p} d \mu\right\}
$$

where the infimum is taken over all $A M$-admissible sequences $\left(\rho_{i}\right)$ for $\Gamma$.

Since the space $X$ is proper, instead of admissible Borel functions, it is possible to use lower semicontinuous non-negative functions as admissible for the $M_{p^{-}}$and $A M_{p^{-}}$-moduli (see, e.g., [5, Proposition $7.14])$.

For the following lemma, we refer to [8], [12] and [9] for the properties of the $A M_{p}$-modulus and to [2], [6] and [1] for those of the $M_{p}$-modulus, $p \geq 1$.

Lemma 2.1. The $A M_{p^{-}}$and $M_{p}$-moduli are outer measures in the set of all paths in $X$, i.e.

(a) $A M_{p}(\emptyset)=0$,

(b) $\Gamma_{1} \subset \Gamma_{2} \Longrightarrow A M_{p}\left(\Gamma_{1}\right) \leq A M_{p}\left(\Gamma_{2}\right)$,

(c) $\Gamma=\bigcup_{j=1}^{\infty} \Gamma_{j} \Longrightarrow A M_{p}(\Gamma) \leq \sum_{i=j}^{\infty} A M_{p}\left(\Gamma_{j}\right)$,

(d) $A M_{1}(\Gamma) \leq M_{1}(\Gamma)$ and $A M_{p}(\Gamma)=M_{p}(\Gamma), p>1$, for every path family $\Gamma$.

The properties (a)-(c) also hold for the $M_{p}$-modulus.

We employ the following notation for path families associated with an arbitrary set $E \subset X$ and an open bounded set $G \supset E$ :

$$
\Gamma(E, G)=\Gamma(E) \cap \Gamma(X \backslash G) \text { and } \Gamma(E)=\{\gamma: \gamma \text { meets } E\} .
$$

\section{3. $\mathrm{Cap}_{\mathrm{p}}^{\mathrm{M}}$-capacity}

In this section, we assume that $X$ is a proper metric space with a Borel regular measure $\mu$ and introduce a new capacity for the condenser $(E, G)$, where $E$ is an arbitrary subset of a bounded open set $G$ in $X$. Since a metric space usually has plenty of Lipschitz functions but need not contain many curves, such a capacity is not possible without an assumption that guarantees plenitude of curves, and we use the quasiconvexity property of $X$, i.e. there is $c<\infty$ such that for all $x, y \in X, x \neq y$, there exists a path $\gamma$ joining $x$ to $y$ whose length satisfies $\ell(\gamma) \leq c d(x, y)$. Note that we do not need the quasiconvexity property for $G$ but for $X$.

A complete doubling $p$-Poincaré space $X$ is quasiconvex, see [2, Chapter 4], but the converse is not true as simple examples show.

We mostly work with Lipschitz functions in $X$. For such a function $u$, a non-negative Borel function $g$ is an upper gradient of $u$ in $X$ if for every path $\gamma$ in $X$,

$$
|u(\gamma(\ell))-u(\gamma(0))| \leq \int_{\gamma} g d s
$$


(see [2, Chapters 1-2] for the properties of functions and their upper gradients). The lower pointwise dilatation

$$
|\nabla u(x)|=\liminf _{r \rightarrow 0} \sup _{y \in B(x, r)} \frac{|u(y)-u(x)|}{r}
$$

is an upper gradient of $u$, see [2, Proposition 1.14]. In $\mathbb{R}^{n},|\nabla u(x)|$ is a unique minimal upper gradient for a Lipschitz function $u$, see [2, Examples A1].

Let $G$ be a fixed bounded open set in $X$, and $E$ an arbitrary subset of $G$. An increasing sequence $\left(u_{i}\right)$ of non-negative Lipschitz functions in $X$ is called admissible, $\left(u_{i}\right) \in \operatorname{Ad}(E, G)$, for the condenser $(E, G)$ if $u_{i}=0$ in $X \backslash G$, and

$$
\liminf _{i \rightarrow \infty} u_{i}(x) \geq 1
$$

for $x \in E$. For $p \geq 1$, we define

$$
\operatorname{Cap}_{\mathrm{p}}^{\mathrm{M}}(E, G)=\inf \left\{\liminf _{i \rightarrow \infty} \int_{G} g_{i}^{p} d \mu:\left(u_{i}\right) \in A d(E, G) \text { and } g_{i} \text { is an upper gradient of } u_{i}\right\} .
$$

It is obvious that the $\mathrm{Cap}_{\mathrm{p}}^{\mathrm{M}}$-capacity is monotone, i.e.

$$
E_{1} \subset E_{2} \subset G \Longrightarrow \operatorname{Cap}_{\mathrm{p}}^{\mathrm{M}}\left(E_{1}, G\right) \leq \operatorname{Cap}_{\mathrm{p}}^{\mathrm{M}}\left(E_{2}, G\right) .
$$

In the rest of this section, we assume that $X$ is proper and quasiconvex, and $G \subset X$ is a bounded open set.

Theorem 3.1. If $E$ is an arbitrary subset of $G$, then, for $p \geq 1$,

$$
A M_{p}(\Gamma(E, G)) \leq \operatorname{Cap}_{\mathrm{p}}^{\mathrm{M}}(E, G) \leq M_{p}(\Gamma(E ; G)) .
$$

For $p>1$, the above inequalities are equalities and, in particular,

$$
\operatorname{Cap}_{\mathrm{p}}^{\mathrm{M}}(E, G)=M_{p}(\Gamma(E ; G)) .
$$

Proof. The first inequality $\leq$ in $(3.2)$ is classical. For completeness, we recall the proof. Let $\left(u_{i}\right)$ be an $A d(E, G)$-admissible sequence and $g_{i}$ an upper gradient of $u_{i}$. Now $\left(g_{i}\right)$ is an $A M$-admissible sequence for $\Gamma(E, G)$ because for each path $\gamma \in \Gamma(E, G)$ with $\gamma(\ell) \in E$,

$$
\liminf _{i \rightarrow \infty} \int_{\gamma} g_{i} d s \geq \liminf _{i \rightarrow \infty} u_{i}(\gamma(\ell)) \geq 1
$$

Hence,

$$
A M_{p}(\Gamma(E, G)) \leq \liminf _{i \rightarrow \infty} \int_{G} g_{i}^{p} d \mu
$$

and since this holds for all sequences $\left(u_{i}\right) \in A d(E, G)$ and all upper gradients $g_{i}$ of $u_{i}$, the left-hand side of (3.2) follows.

For the second inequality in (3.2), we use a modification of the method in [2, Lemmata 5.25 and 5.26]. Let $\tilde{\rho}$ be a lower semicontinuous $M$-admissible function for $\Gamma(E, G)$. We may assume that $\tilde{\rho}=0$ in $X \backslash G$ and

$$
\int_{G} \tilde{\rho}^{p} d \mu<\infty
$$


Let $\tau>0$ and set $\rho=\tilde{\rho}+\tau$ in $X$. Now $\rho$ is lower semicontinuous in $X$ and, since $X$ is proper, there is an increasing sequence of continuous functions $\rho_{i}: X \rightarrow[0, \infty)$ such that

$$
\lim _{i \rightarrow \infty} \rho_{i}(x)=\rho(x)
$$

for every $x \in X$. We may assume that $\rho_{i} \geq \tau$ in $X$.

For each $i$, define

$$
u_{i}(x)=\inf \left\{\int_{\gamma} \rho_{i} d s: \gamma \text { joins } X \backslash G \text { to } x\right\}
$$

for $x \in G$ and $u_{i}(x)=0$ for $x \in X \backslash G$. Note that each path $\gamma$ that meets $X \backslash G$ and $x \in G$ has a subpath meeting $X \backslash G$ at $\gamma(0)$ only. Hence, in the definition of $u_{i}(x), x \in G$, we can consider only the paths $\gamma$ that lie in $G$ except at $\gamma(0)$. The sequence $\left(u_{i}\right)$ is increasing and we show that each $u_{i}$ is an $C_{i} c$-Lipschitz function, where $C_{i}=\sup \left\{\rho_{i}(x): x \in X\right\}$ and $c$ is the quasigeodesic constant of $X$. Consider first the case where $x, y \in G$. By symmetry, we may assume $u_{i}(y) \geq u_{i}(x)$. Let $\varepsilon>0$ and choose a path $\gamma_{x y}$ joining $x$ to $y$ with $\ell\left(\gamma_{x y}\right) \leq c d(x, y)$. By the definition of $u_{i}(x)$, there is a path $\gamma_{x}$ from $X \backslash G$ to $x$ such that

$$
u_{i}(x)>\int_{\gamma_{x}} \rho_{i} d s-\varepsilon .
$$

Joining the paths $\gamma_{x}$ and $\gamma_{x y}$ together, we obtain a path $\gamma$ from $X \backslash G$ to $y$. Now

$$
u_{i}(y)-u_{i}(x) \leq \int_{\gamma} \rho_{i} d s-\int_{\gamma_{x}} \rho_{i} d s+\varepsilon \leq \int_{\gamma_{x y}} \rho_{i} d s+\varepsilon \leq C_{i} c d(x, y)+\varepsilon
$$

and, letting $\varepsilon \rightarrow 0$, we obtain the required Lipschitz bound for $u_{i}$. If $x \in X \backslash G$ and $y \in G$, then $u_{i}(x)=0$, and choosing $\gamma_{x y}$ as before, we have

$$
u_{i}(y)-u_{i}(x)=u_{i}(y) \leq \int_{\gamma_{x y}} \rho_{i} d s \leq C_{i} c d(x, y) .
$$

For $y, x \in X \backslash G$, the inequality is trivial.

The function $\rho_{i}$ is an upper gradient of $u_{i}$. If $\gamma$ is a path joining $y$ and $x$ which lie in $G$, then, by symmetry, we can assume that $u_{i}(y) \geq u_{i}(x)$, and for $\varepsilon>0$ we can choose a path $\gamma_{x}$ joining $X \backslash G$ to $x$ such that

$$
u_{i}(x)>\int_{\gamma_{x}} \rho_{i} d s-\varepsilon ;
$$

and joining the paths $\gamma_{x}$ and $\gamma$ together, we obtain the path $\tilde{\gamma}$ joining $X \backslash G$ to $y$. Thus

$$
u_{i}(y)-u_{i}(x) \leq \int_{\tilde{\gamma}} \rho_{i} d s-\int_{\gamma_{x}} \rho_{i} d s+\varepsilon=\int_{\gamma} \rho_{i} d s+\varepsilon
$$

and letting $\varepsilon \rightarrow 0$, we obtain the required inequality. If $y \in X \backslash G, x \in G$, and $\gamma$ is a path joining $y$ to $x$, then

$$
u_{i}(y)-u_{i}(x)=u_{i}(y) \leq \int_{\gamma} \rho_{i} d s .
$$

The case $x, y \in X \backslash G$ is again trivial.

Next let $\varepsilon>0$ and

$$
E_{\varepsilon}=\left\{x \in E: \lim _{i \rightarrow \infty} u_{i}(x)<1-\varepsilon\right\} .
$$


Fix $x \in E_{\varepsilon}$. Then there is a sequence of paths $\gamma_{i}$ from $X \backslash G$ to $x$ such that for each $i$

$$
1-\varepsilon>u_{i}(x) \geq \int_{\gamma_{i}} \rho_{i} d s-2^{-i} \varepsilon .
$$

Now for $j \geq i$

$$
u_{i}(x) \leq u_{j}(x) \leq \int_{\gamma_{j}} \rho_{j} d s
$$

Let

$$
L=\liminf _{i \rightarrow \infty} \ell\left(\gamma_{i}\right)
$$

and reparameterize the paths $\gamma_{i}$ as $\tilde{\gamma}_{i}(t)=\gamma_{i}\left(t \ell\left(\gamma_{i}\right)\right), t \in[0,1]$. Since we may assume that each $\gamma_{i}$ meets $X \backslash G$ at $\gamma_{i}(0)$ only,

$$
\ell\left(\gamma_{i}\right) \leq \int_{\gamma_{i}} \frac{\rho_{i}}{\tau} d s \leq \frac{1-\varepsilon}{\tau}<\frac{1}{\tau}
$$

and so the curves $\tilde{\gamma}_{i}$ are $\ell\left(\gamma_{i}\right)$-Lipschitz and uniformly $1 / \tau$-Lipschitz and thus an equicontinuous family of mappings from $[0,1]$ to the compact space $\bar{G}$. By the Ascoli theorem there is a subsequence of $\left(\tilde{\gamma}_{i}\right)$, denoted again by $\left(\tilde{\gamma}_{i}\right)$, which converges uniformly to a $1 / \tau$-Lipschitz curve $\tilde{\gamma}:[0,1] \rightarrow \bar{G}$. Clearly, $\tilde{\gamma}(0) \in X \backslash G$ and $\tilde{\gamma}(1)=x$, and by the continuity of $\rho_{j}$ in $\bar{G}$,

$$
\lim _{i \rightarrow \infty} \rho_{j}\left(\tilde{\gamma}_{i}(t)\right)=\rho_{j}(\tilde{\gamma}(t))
$$

for each $j$ and $t \in[0,1]$.

Next let $\gamma$ be the reparametrization of $\tilde{\gamma}$ by arch length and note that

$$
\ell(\gamma) \leq \liminf _{i \rightarrow \infty} \ell\left(\gamma_{i}\right)=L
$$

Denote the length function of $\tilde{\gamma}_{i}$ as

$$
s_{i}(t)=\ell\left(\tilde{\gamma}_{i} \mid[0, t]\right), t \in[0,1]
$$

and the length function of $\tilde{\gamma}$ as

$$
s(t)=\ell(\tilde{\gamma} \mid[0, t]), t \in[0,1] .
$$

Now $s_{i}^{\prime}(t)=\ell\left(\gamma_{i}\right)$ for $t \in(0,1)$, and at the point $t_{0} \in(0,1)$ of the differentiability of $s$, we have for $t_{0}<t_{1} \leq 1$

$$
\begin{aligned}
& \frac{s\left(t_{1}\right)-s\left(t_{0}\right)}{t_{1}-t_{0}}=\frac{\ell\left(\tilde{\gamma} \mid\left[t_{0}, t_{1}\right]\right)}{t_{1}-t_{0}} \leq \liminf _{i \rightarrow \infty} \frac{\ell\left(\tilde{\gamma}_{i} \mid\left[t_{0}, t_{1}\right]\right)}{t_{1}-t_{0}} \\
= & \frac{1}{t_{1}-t_{0}} \liminf _{i \rightarrow \infty}^{t_{1}} \int_{t_{0}}^{t_{1}} s_{i}^{\prime}(t) d t=\liminf _{i \rightarrow \infty} \frac{\ell\left(\gamma_{i}\right)\left(t_{1}-t_{0}\right)}{t_{1}-t_{0}}=L
\end{aligned}
$$

and hence $s^{\prime}(t) \leq L$ for a.e. $t \in[0,1]$. For $j \geq i$, we have

$$
\int_{\tilde{\gamma}_{j}} \rho_{j} d s \geq \int_{\tilde{\gamma}_{j}} \rho_{i} d s=\int_{0}^{1} \rho_{i}\left(\tilde{\gamma}_{j}(t)\right) s_{j}^{\prime}(t) d t=\ell\left(\gamma_{j}\right) \int_{0}^{1} \rho_{i}\left(\tilde{\gamma}_{j}(t)\right) d t .
$$

Note that the function $s$ is absolutely continuous because $\tilde{\gamma}$ is a Lipschitz curve. Now (3.4), (3.5), and the continuity of $\rho_{i}$ yield for each $i$

$$
1-\varepsilon \geq \liminf _{j \rightarrow \infty} \int_{\tilde{\gamma}_{j}} \rho_{j} d s \geq L \int_{0}^{1} \rho_{i}(\tilde{\gamma}(t)) d t \geq \int_{0}^{1} \rho_{i}(\tilde{\gamma}(t)) s^{\prime}(t) d t=\int_{\gamma} \rho_{i} d s .
$$


This leads to a contradiction since, by the Lebesgue increasing convergence theorem,

$$
\lim _{i \rightarrow \infty} \int_{\gamma} \rho_{i} d s=\int_{\gamma} \rho d s \geq \int_{\gamma} \tilde{\rho} d s \geq 1
$$

for every path $\gamma \in \Gamma(E, G)$ and thus

$$
\lim _{i \rightarrow \infty} u_{i}(x) \geq 1-\varepsilon
$$

for each $x \in E$.

Now $\left(u_{i} /(1-\varepsilon)\right)$ is an admissible sequence for $\operatorname{Cap}_{\mathrm{p}}^{\mathrm{M}}(E, G)$ and, by the Lebesgue bounded convergence theorem,

$$
\operatorname{Cap}_{\mathrm{p}}^{\mathrm{M}}(E, G) \leq \liminf _{i \rightarrow \infty} \int_{G}\left(\frac{\rho_{i}}{1-\varepsilon}\right)^{p} d \mu=\int_{G}\left(\frac{\tilde{\rho}+\tau}{1-\varepsilon}\right)^{p} d \mu
$$

and, since $\tilde{\rho}$ is an arbitrary $M$-admissible function for $\Gamma(E, G)$, letting $\varepsilon \rightarrow 0$ and $\tau \rightarrow 0$, we complete the proof for the right inequality of (3.2).

Because $A M_{p}(\Gamma(E, G))=M_{p}(\Gamma(E, G))$ for $p>1$, the equality case in (3.2) is clear.

Lemma 3.1. If $E \subset G$ is an arbitrary set and $p \geq 1$, then there is a Borel set $E^{\prime} \supset E$ such that

$$
\operatorname{Cap}_{\mathrm{p}}^{\mathrm{M}}\left(E^{\prime}, G\right)=\operatorname{Cap}_{\mathrm{p}}^{\mathrm{M}}(E, G) .
$$

Proof. If $\operatorname{Cap}_{\mathrm{p}}^{\mathrm{M}}(E, G)=\infty$, we can choose $E^{\prime}=G$. Then the monotonicity of Cap $\mathrm{p}$ implies

$$
\operatorname{Cap}_{\mathrm{p}}^{\mathrm{M}}(G, G) \geq \operatorname{Cap}_{\mathrm{p}}^{\mathrm{M}}(E, G)=\infty .
$$

Suppose that $\operatorname{Cap}_{\mathrm{p}}^{\mathrm{M}}(E, G)<\infty$ and for each $j \in \mathbb{N}$ choose an $\operatorname{Ad}(E, G)$ admissible sequence $\left(u_{i}^{j}\right)$ such that

$$
\operatorname{Cap}_{\mathrm{p}}^{\mathrm{M}}(E, G) \geq \liminf _{i \rightarrow \infty} \int_{G}\left(g_{i}^{j}\right)^{p} d \mu-1 / j
$$

where $g_{i}^{j}$ is an upper gradient of $u_{i}^{j}$.

Now the set

$$
F^{j}=\left\{x \in G: \liminf _{i \rightarrow \infty} u_{i}^{j}(x) \geq 1\right\}
$$

is a Borel set and $F^{j} \supset E$. The set $E^{\prime}=\cap_{j} F^{j}$ is a Borel set and contains $E$ so that $\operatorname{Cap}_{p}\left(E^{\prime}, G\right) \geq$ $\operatorname{Cap}_{p}(E, G)$. Using (3.6), we obtain the converse inequality

$$
\begin{gathered}
\operatorname{Cap}_{p}\left(E^{\prime}, G\right) \leq \liminf _{j \rightarrow \infty} \liminf _{i \rightarrow \infty} \int_{G}\left(g_{i}^{j}\right)^{p} d \mu \\
\leq \liminf _{j \rightarrow \infty}\left(\operatorname{Cap}_{\mathrm{p}}^{\mathrm{M}}(E, G)+1 / j\right)=\operatorname{Cap}_{\mathrm{p}}^{\mathrm{M}}(E, G) .
\end{gathered}
$$

From Theorem 3.1 and Lemma 3.1, we obtain:

Corollary 3.1. If $p>1$, then for each set $E \subset G$, there is a Borel set $E^{\prime} \supset E$ such that $M_{p}(\Gamma(E, G))=$ $M_{p}\left(\Gamma\left(E^{\prime}, G\right)\right)$.

Since $\operatorname{Cap}_{\mathrm{p}}^{\mathrm{M}}(E, G)=M_{p}(\Gamma(E, G))$ for $p>1$, the $\operatorname{Cap}_{\mathrm{p}}^{\mathrm{M}}(E, G)$-capacity for all sets $E \subset G$ inherits all the properties of the $M_{p}$-modulus in a quasiconvex space $X$. For the properties of the Choquet capacity, see Section 4. 
Theorem 3.2. The $\mathrm{Cap}_{\mathrm{p}}^{\mathrm{M}}$-capacity, $p>1$, has the following properties:

(a) $\mathrm{Cap}_{\mathrm{p}}^{\mathrm{M}}$ is subadditive. i.e. if $E_{i} \subset G, i=1,2, \ldots$, then

$$
\operatorname{Cap}_{\mathrm{p}}^{\mathrm{M}}\left(\bigcup_{i} E_{i}, G\right) \leq \sum_{i} \operatorname{Cap}_{\mathrm{p}}^{\mathrm{M}}\left(E_{i}, G\right) .
$$

(b) If $K_{1} \supset K_{2} \supset \ldots$ are compact sets in $G$, then

$$
\lim _{i \rightarrow \infty} \operatorname{Cap}_{\mathrm{p}}^{\mathrm{M}}\left(K_{i}, G\right)=\operatorname{Cap}_{\mathrm{p}}^{\mathrm{M}}\left(\bigcap_{i} K_{i}, G\right) .
$$

(c) $\mathrm{Cap}_{\mathrm{p}}^{\mathrm{M}}$ is a Choquet capacity, i.e. for a Suslin set $E \subset G$,

$$
\operatorname{Cap}_{\mathrm{p}}^{\mathrm{M}}(E, G)=\sup \left\{\operatorname{Cap}_{\mathrm{p}}^{\mathrm{M}}(K, G): K \subset E \text { compact }\right\} .
$$

Proof. The subadditivity of the $M_{p}$-modulus is well known, see Lemma 2.1, and hence Theorem 3.1 implies (a). For (b), let $K=\cap_{i} K_{i}$ and note that by the monotonicity

$$
\lim _{i \rightarrow \infty} \operatorname{Cap}_{\mathrm{p}}^{\mathrm{M}}\left(K_{i}, G\right) \geq \operatorname{Cap}_{\mathrm{p}}^{\mathrm{M}}(K, G) .
$$

For the reverse inequality, let $\varepsilon>0$ and choose a sequence $\left(u_{i}\right) \in A d(K, G)$ such that

$$
\operatorname{Cap}_{\mathrm{p}}^{\mathrm{M}}(K, G) \geq \liminf _{i \rightarrow \infty} \int_{G} g_{i}^{p} d \mu-\varepsilon .
$$

The function $u=\lim _{i} u_{i}$ is lower semicontinuous in $G$ as a limit of an increasing sequence of continuous functions $u_{i}$. Thus the set $U=\{x \in G: u(x)>1-\varepsilon\}$ is open and contains $K$. Now there is $i_{0}$ such that $K_{i} \subset U$ for $i \geq i_{0}$ and thus $\left(u_{i} /(1-\varepsilon)\right) \in A d\left(K_{i}, G\right]$ for $i \geq i_{0}$. By (3.7),

$$
\lim _{i \rightarrow \infty} \operatorname{Cap}_{\mathrm{p}}^{\mathrm{M}}\left(K_{i}, G\right) \leq \liminf _{i \rightarrow \infty} \int_{G}\left(\frac{g_{i}}{1-\varepsilon}\right)^{p} d \mu \leq \frac{\operatorname{Cap}_{\mathrm{p}}^{\mathrm{M}}(K, G)+\varepsilon}{(1-\varepsilon)^{p}}
$$

and letting $\varepsilon \rightarrow 0$, we obtain (b).

The map $E \mapsto \operatorname{Cap}_{\mathrm{p}}^{\mathrm{M}}(E, G)$ is monotone and satisfies (a) and (b). Hence, by the Choquet capacitibility theorem, see [4], it satisfies (c).

\section{Dirichlet capacity}

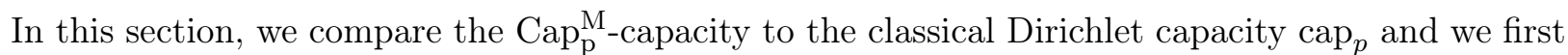
recall its definition and basic properties due to G. Choquet. Originally, this capacity used $C_{0}^{\infty}$-functions in $\mathbb{R}^{n}$ and their gradients, but the upper gradients for Lipschitz functions work as well in a metric measure space $X$, see [2, Section 6.3].

We again assume that $X$ is a proper quasigeodesic space and $G \subset X$ is a fixed bounded open set.

Let $K$ be a compact subset of $G$, and $A d_{C}(K, G)$ the family of all Lipschitz functions such that $u \geq 1$ in $K$ and $u=0$ in $X \backslash G$. Define

$$
\operatorname{cap}_{p}(K, G)=\inf \left\{\int_{G} g^{p} d \mu: u \in A d_{C}(K, G), g \text { an upper gradient of } u\right\}
$$

Obviously, the infimum does not change if restricted to test functions satisfying $0 \leq u \leq 1$. The condition that a test function $u \in A d_{C}(K, G)$ satisfies $u=0$ in $X \backslash G$ can be replaced, due to the continuity of $u$, by the requirement that $u$ has a compact support in $G$. 
If $U \subset G$ is open, then we set

$$
\operatorname{cap}_{p}(U, G)=\sup \left\{\operatorname{cap}_{p}(K, G): K \subset U \text { compact }\right\}
$$

and, for an arbitrary set $E \subset G$,

$$
\operatorname{cap}_{p}(E, G)=\inf \left\{\operatorname{cap}_{p}(U, G): U \text { open, } E \subset U \subset G\right\} .
$$

Now there are two definitions for $\operatorname{cap}_{p}(E, G)$ when $E$ is compact, but since the competitors are continuous, the both definitions give the same value.

The cap $_{p}$-capacity, $p \geq 1$, has the following properties:

(i) monotonicity: $E_{1} \subset E_{2} \Longrightarrow \operatorname{cap}\left(E_{1}, G\right) \leq \operatorname{cap}_{p}\left(E_{2}, G\right)$;

(ii) subadditivity: $E_{1} \subset E_{2} \subset \ldots \Longrightarrow \lim _{i} \operatorname{cap}_{p}\left(E_{i}, G\right)=\operatorname{cap}_{p}\left(\bigcup_{i} E_{i}\right)$;

(iii) $K_{1} \supset K_{2} \supset \ldots$ compact $\Longrightarrow \lim _{i} \operatorname{cap}_{p}\left(K_{i}, G\right)=\operatorname{cap}_{p}\left(\bigcap_{i} K_{i}, G\right)$.

By the Choquet capacibility theorem, for all Suslin sets $E \subset G$,

$$
\operatorname{cap}_{p}(E, G)=\sup \left\{\operatorname{cap}_{p}(K, G): K \subset E \text { compact }\right\} .
$$

For the Choquet theory, see [4] and [3], and for the Dirichlet capacity in $X$, see [2, Section 6.3].

We frequently use the following lemma:

Lemma 4.1. For $p \geq 1$ and $K \subset G$ compact, the equality

$$
\operatorname{cap}_{p}(K, G)=M_{p}(\Gamma(K, G))=A M_{p}(\Gamma(K, G))=\operatorname{Cap}_{\mathrm{p}}^{\mathrm{M}}(K, G)
$$

holds.

Proof. For $p=1$, by [11, Lemma 3.3],

$$
\operatorname{cap}_{1}(K, G)=M_{1}(\Gamma(K, G))=A M_{1}(\Gamma(K, G))
$$

and thus, by Theorem 3.1, this equality holds in (4.1). For $p>1,(4.1)$ is well known, see e.g., [2, Chapter 5] and [1]. Note that for $p>1, M_{p}(\Gamma)=A M_{p}(\Gamma)$ for every path family $\Gamma$ in $X$, see [11, Lemma 3.3].

Lemma 4.2. If $E \subset G$ is an arbitrary set and $p \geq 1$, then

$$
\operatorname{Cap}_{\mathrm{p}}^{\mathrm{M}}(E, G) \geq \operatorname{cap}_{p}(E, G) .
$$

Proof. Choose a Borel set $E^{\prime} \supset E$ such that $\operatorname{Cap}_{\mathrm{p}}^{\mathrm{M}}(E, G)=\operatorname{Cap}_{\mathrm{p}}^{\mathrm{M}}\left(E^{\prime}, G\right)$. Then, by Lemma 4.1 and the Choquet capacibility theorem,

$$
\begin{aligned}
& \operatorname{Cap}_{\mathrm{p}}^{\mathrm{M}}(E, G)=\operatorname{Cap}_{\mathrm{p}}^{\mathrm{M}}\left(E^{\prime}, G\right) \geq \sup _{\left\{K \subset E^{\prime} \text { compact }\right\}} \operatorname{Cap}_{\mathrm{p}}^{\mathrm{M}}(K, G) \\
& =\sup _{\left\{K \subset E^{\prime} \text { compact }\right\}} \operatorname{cap}_{p}(K, G)=\operatorname{cap}_{p}\left(E^{\prime}, G\right) \geq \operatorname{cap}_{p}(E, G) .
\end{aligned}
$$

Lemma 4.3. Suppose that $E$ is an arbitrary subset of $G$. For $p>1$,

$$
A M_{p}(\Gamma(E, G))=M_{p}(\Gamma(E, G)) \leq \operatorname{cap}_{p}(E, G),
$$

and for $p=1$,

$$
A M_{1}(\Gamma(E, G)) \leq \operatorname{cap}_{1}(E, G) .
$$


Proof. The first equality in (4.3) is due to the fact that $A M_{p}=M_{p}$ for $p>1$. For the inequality in (4.3), let $U \subset G$ be an open set with $U \supset E$ and choose compact sets $K_{1} \subset K_{2} \subset \ldots \subset U$ such that $\cup_{i} K_{i}=U$. Now $\Gamma\left(K_{i}, G\right) \subset \Gamma\left(K_{i+1}, G\right)$ and

$$
\bigcup_{i} \Gamma\left(K_{i}, G\right)=\Gamma(U, G)
$$

and, since $p>1$, we have $M_{p}(\Gamma(U, G))=\lim _{i \rightarrow \infty} M_{p}\left(\Gamma\left(K_{i}, G\right)\right)$, see [1]. Then, from (4.1), it follows that

$$
M_{p}(\Gamma(U, G))=\lim _{i \rightarrow \infty} M_{p}\left(\Gamma\left(K_{i}, G\right)\right)=\lim _{i \rightarrow \infty} \operatorname{cap}_{p}\left(K_{i}, G\right)=\operatorname{cap}_{p}(U, G) .
$$

Since this holds for all open sets $U \supset E$,

$$
\begin{aligned}
& M_{p}(\Gamma(E, G)) \leq \inf _{G \supset U \supset E} M_{p}(\Gamma(U, G)) \\
= & \inf _{G \supset U \supset E} \operatorname{cap}_{p}(\Gamma(U, G))=\operatorname{cap}_{p}(E, G) .
\end{aligned}
$$

For (4.4), we can proceed as above but now, by [8, Lemma 3.11], we have

$$
\operatorname{cap}_{1}(U, G)=\lim _{i \rightarrow \infty} \operatorname{cap}_{1}\left(K_{i}, G\right)=\lim _{i \rightarrow \infty} M_{1}\left(\Gamma\left(K_{i}, G\right)\right) \geq A M_{1}(\Gamma(U, G))
$$

and hence, for every open set $U \supset E$,

$$
\operatorname{cap}_{1}(U, G) \geq A M_{1}(\Gamma(U, G)) \geq A M_{1}(\Gamma(E, G)) .
$$

The following summarizes the situation with Suslin sets for $p=1$.

Lemma 4.4. If $E \subset G$ is a Suslin set, then

$$
\operatorname{cap}_{1}(E, G)=A M_{1}(\Gamma(E, G)) \leq \operatorname{Cap}_{1}^{\mathrm{M}}(E, G) \leq M_{1}(\Gamma(E, G)) .
$$

Proof. To prove the first equality in (4.5), it suffices to show, by (4.4), that

$$
\operatorname{cap}_{1}(E, G) \leq A M_{1}(\Gamma(E, G)) .
$$

Since $E$ is a Suslin set, the Choquet capacibility theorem yields

$$
\operatorname{cap}_{1}(E, G)=\sup \left\{\operatorname{cap}_{1}(K, G): K \subset E \text { compact }\right\},
$$

and for each compact set $K \subset E$,

$$
\operatorname{cap}_{1}(K, G)=A M_{1}(\Gamma(K, G)) \leq A M_{1}(\Gamma(E, G))
$$

and (4.6) follows. The rest follows from Theorem 3.1 and Lemma 4.2.

The following theorem summarizes the situation for $p>1$ and $p=1$.

Theorem 4.1. If $X$ is a proper quasigeodesic metric space, $G \subset X$ a bounded open set, and $E \subset G$ an arbitrary set, then, for $p>1$,

$$
A M_{p}(\Gamma(E, G))=\operatorname{cap}_{p}(E, G)=\operatorname{Cap}_{\mathrm{p}}^{\mathrm{M}}(E, G)=M_{p}(\Gamma(E, G)),
$$

and for $p=1$,

$$
A M_{1}(\Gamma(E, G)) \leq \operatorname{cap}_{1}(E, G) \leq \operatorname{Cap}_{1}^{\mathrm{M}}(E, G) \leq M_{1}(\Gamma(E, G)) .
$$


Proof. Since $A M_{p}(\Gamma(E, G))=M_{p}(\Gamma(E, G))$, the proof for (4.7) in the case $p>1$ follows from Theorem 3.1, as well as Lemmata 4.2 and 4.3. The inequalities in (4.8) follow from Theorem 3.1, (4.4), and (4.2).

\section{REFERENCES}

1. L. Ambrosio, S. Di Marino, and G. Savaré, "On the duality between p-modulus and probability measures," J. Eur. Math. Soc., 17, 1817-1853 (2015).

2. A. Björn and J. Björn, Nonlinear potential theory on metric spaces, EMS Tracts Math., vol. 17, European Mathematical Society (EMS), Zurich, 2011.

3. M. Brelot, Lectures on potential theory. Notes by K. N. Gowrisankaran and M. K. Venkatesha Murthy, second edition, revised and enlarged with the help of S. Ramaswamy, Tata Institute of Fundamental Research Lectures on Mathematics, N 19 Tata Institute of Fundamental Research, Bombay, 1967.

4. G. Choquet, "Theory of capacities," Ann. Inst. Fourier, 5, 131-295 (1953-54).

5. G. B. Folland, Real analysis, 2nd ed., Wiley, New York, 1999.

6. B. Fuglede, "Extremal length and functional completion," Acta Math., 98, 171-219 (1957).

7. J. Heinonen, P. Koskela, N. Shanmugalingam, and J. Tyson, Sobolev spaces on metric measure spaces, Cambridge University Press, 2015.

8. V. Honzlová-Exnerová, O. Kalenda, J. Malý, and O. Martio, "Plans on measures and AM-modulus," J. Funct. Anal., preprint arXiv 1904.04527 (to appear).

9. V. Honzlová-Exnerová, J. Malý, and O. Martio, "Modulus in Banach function spaces," Ark. Mat., 55(1), 105-130 (2017).

10. V. Honzlová-Exnerová, J. Malý, and O. Martio, "Functions of bounded variation and the $A M$-modulus in $\mathbb{R}^{n}, "$ Nonlinear Anal., 177, 553-571 (2018).

11. V. Honzlová-Exnerová, O. Kalenda, J. Malý, and O. Martio, " $A M$-modulus and Hausdorff measure of codimension one in metric measure spaces," Math. Nachr. (to appear).

12. O. Martio, "Functions of bounded variation and curves in metric measure spaces," Adv. Calc. Var., 9(4), 305-322 (2016).

13. N. Shanmugalingam, "Newtonian spaces: An extension of Sobolev spaces to metric measure spaces," Rev. Mat. Iberoam., 16, 243-279 (2000).

\section{Olli Martio}

Department of Mathematics and Statistics, FI-00014 University of Helsinki, Finland

E-Mail: olli.martio@helsinki.fi 